\title{
Annotazioni Critiche su Aquila e Priscilla, il Titulius Priscae e le Catacombe di Priscilla a Roma
}

\author{
ILARIA RANELLI *
}

\begin{abstract}
The article proposes an analysis of the tradition concerning Aquila and Priscilla; the titulus Priscae in Rome is probably connected not with Aquila's wife, but with the later Virgin and Martyr St. Prisca. We don't know whether the Aquila and the Priscilla who were buried in the catacombs of Priscilla can be identified with St. Paul's friends, but it is sure that these catacombs were possessed -at a very early date- by the Acilii Glabriones. They contain inscriptions from the IInd century $A D$ onward, but perhaps there were Christian members of the family already in the Ist century: one of the Acilii Glabriones was a Roman senator who was put to death during Domitian's persecution; and it is highly probable that he was a Christian.

\section{ABRÉGÉ}

L'étude analyse en premier lieu la tradition sur Aquila et Priscille.

Probablement le titulus Priscae est lié non avec la femme d'Aquila, mais avec la plus tarde Vierge et Martyr Prisque. Nous ne savons pas si les Aquila et Priscilla ensevelis dans le cimetière de Priscilla sont identifiables avec les amis de saint Paul, mais il est bien sûr que le cimetière était propriété des Acilii Glabriones dès une très haute antiquité. Dans le même cimetière ont eté découvertes plusieurs inscriptions du llème siècle apr. J.-C., peut-être ainsi du ler. $\dot{A}$ la fin du ler siècle apr. J.-C. un Acilius Glabrio, senateur romain, fut condamné à mort par Domitien, et très probablement il était chrétien.
\end{abstract}

* Universidad de Milán. 


\section{AQUILA E PRISCILLA: LE FONTI NEOTESTAMENTARIE E PATRISTICHE}

Le notizie storiche di cui disponiamo a proposito di Aquila e di Priscilla, i collaboratori di s. Paolo, non sono numerose, ma sono comunque assolutamente attendibili e derivano tutte da fonti neotestamentarie, Luca e Paolo. Aquila era un Giudeo romanizzato provvisto di cognomen latino e originario del Ponto, sposo di Prisca (questa la forma del nome in $R m 16$, 3; II Tim 4, 19) o Priscilla (cosi in / Cor 16, 19; Act 18, 2. 18. 19. 26), la cui provenienza non è certa: in Act 18, 2 solo Aquila è chiamato Giudeo ': questo potrebbe far supporre che Priscilla non fosse tale, anche se non abbiamo mezzi per affermarlo con sicurezza. Fabbricante di tende, come s. Paolo, Aquila era a Roma nel 49, quando ne fu espulso insieme con la moglie in base alla disposizione di Claudio (Suet. Claud. 25) che allontanava tutti i Giudei dall'Urbe (Act. 18, 2-3):

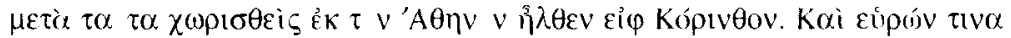

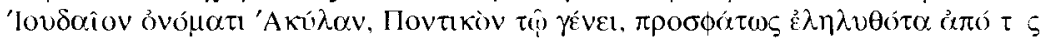

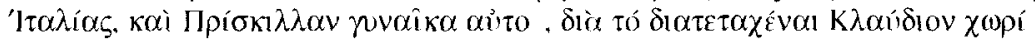

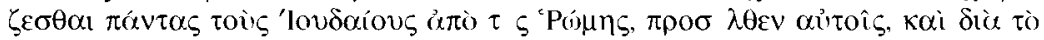

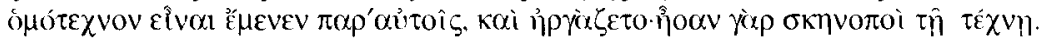

Secondo il Sinassario Costantinopolitano (13 febbraio), i due furono battezzati da Paolo; il Leclercq supponeva invece che essi fossero discepoli di Pietro; la Sordi ${ }^{2}$ pensa che essi siano stati espulsi da Roma esclusivamente in quanto Giudei -o almeno perché Aquila era un Giudeo-, dato che il provvedimento di Claudio colpiva i Giudei e non, formalmente, i Cristiani, e dato che Luca parla di Aquila come di un Giudeo in quel momento e non ancora come di un "fratello»: essi pertanto furono probabilmente convertiti da Paolo quando si rifugiarono a Corinto nel 50-51, dove diedero ospitalità all'apostolo (Act 18, 2-3). Quindi le fonti neotestamentarie li fanno trovare ad Efeso, dove perfezionarono l'istruzione di Apollo e chiamarono Paolo (Act 18, 18-19 e 26) ${ }^{3}$. Fondarono anzi chiese domesti-

M.-F. Baslez, Saint Paul, Paris 1991, tr. it. Paolo di Tarso. L'apostolo delle genti, Torino 1993, pp. 226 e 234; cfr. T. Garcia de Orbiso, Aquila e Priscilla, in Bibliotheca Sanctorum, II, Roma 1962, coll. 326-328; V. Saxer, S.v. Prisca, in Dizionario Patristico e di Antichità Cristiane, II, Casale Monferrato 1983, coll. 1204-1205; Storia dei Santi e della santità cristiana, I, La schiera dei testimoni, a c. di F. Chiovaro, tr. it. Milano 1991, pág. 294.

M. SoRdi, /l Cristianesimo e Roma, Bologna 1965, págs. 63-65.

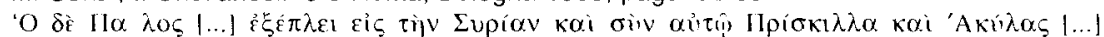

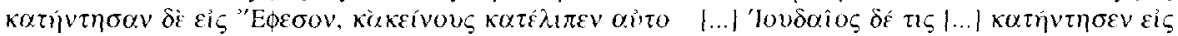

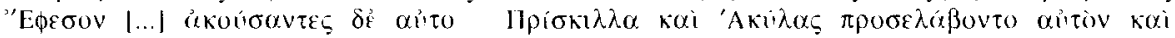

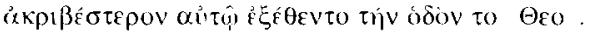




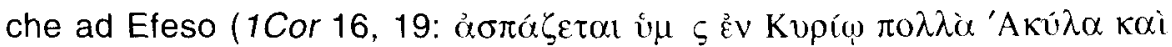

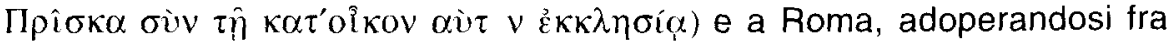
l'altro, non si sa precisamente in quale occasione, a salvare la vita di Paolo

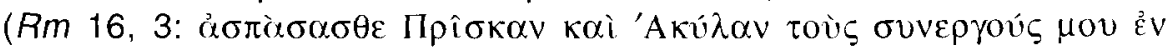

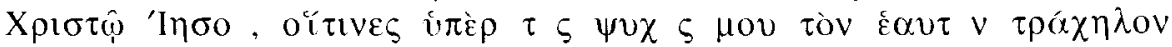

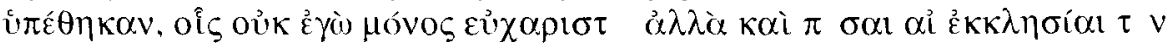

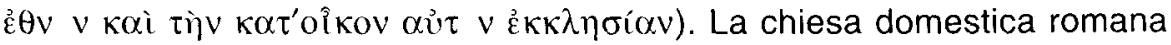
dei due sposi è tradizionalmente identificata con il titulus Priscae sull'Aventino, una identificazione problematica su cui torneremo ${ }^{4}$. Sembra invece fuori discussione oggi il valore probante del passo citato del cap. 16 dell'Epistola ai Romani ai fini dell'attestazione dell'esistenza di una casa di Aquila e Prisca a Roma ${ }^{5}$. Da Roma, i coniugi ritornarono ad Efeso secon-

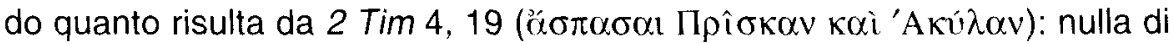
certo ci è dato sapere sulle circostanze e sul luogo della loro morte. II Martyrologium Romanum ricorda Aquila alla data dell's luglio, ma che egli sia morto a Roma, e da martire, non è attestato da altre fonti sicure. Vedremo che nel cimitero di Priscilla furono effettivamente sepolti un Aquila ed una Prisca, ma non è certo che si tratti dei collaboratori di s. Paolo. II Piccolo Martirologio Romano, all'8 luglio, fa morire i due in Asia Minore. Gli Acta Sanctorum ${ }^{6}$ ricordano che di Aquila parla anche il Menologio pridie idus lulii. I Menei greci li riportano al XIII febbraio: 'A

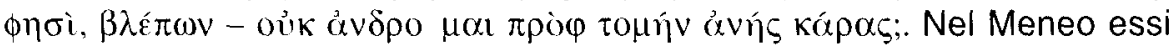
sono presentati come martiri decapitati e facenti parte dei LXX discepoli. Questa tradizione del martirio è viva in ambito greco, come sembra confermare Giovanni Crisostomo, una fonte patristica importante sui due dopo quelle neotestamentarie. Nella Hom. $X X X$ in Epistolam ad Romanos (PG LX 664-65), commentando le parole stesse di Paolo, Giovanni dice: $\pi \rho$ tov

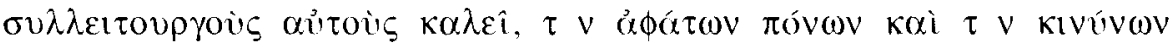

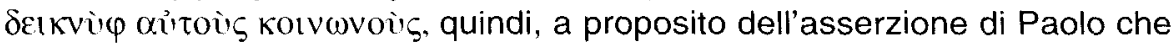
essi «hanno posto la loro testa per lui», Giovanni esclama, facendo notare le difficoltà e i pericoli che $i$ due ebbero ad affrontare sotto Nerone: $\varepsilon \hat{i} \delta \varepsilon \varsigma$

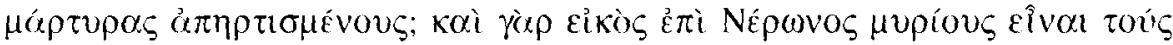
kıvúvouc. Essi sono dunque per Giovanni "perfetti martiri» -o "perfetti testi-

G.B. De Rossi, Della casa d'Aquila sull'Aventino, "Bull. di Archeol. Cristiana" 1867, págs. 44-46; Priscilla e gli Acilii Glabriones, "Bull. di Archeol. Cristiana" 1888-89, págs. 128 sgg.

Per ampia documentazione sui dubbi sollevati in passato si veda $\mathrm{H}$. Leclercq. Prisque in Dictionnaire de Archéologie Chrétienne et de Liturgie, XIV 1, Paris 1939, coll. 1876-1887, part. 1879-1880.

AASS II, lufii, Venezia 1752, págs. 534-37, De SS Aquila et Priscilla coniugibus, forte in Asia Minore, Sylloge Historica. 
moni": ma vedremo subito che la prima interpretazione sembra, per Giovanni, quella giusta, dato che poco dopo il Crisostomo parla del loro

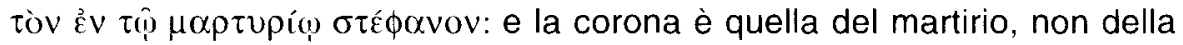
testimonianza. Commentando la gratitudine di Paolo e di tutte le Chiese nei loro confronti per il l'impegno profuso, Giovanni prosegue: $\dot{\varepsilon} v \tau \alpha \quad \theta \alpha \tau \eta \dot{\eta} v$

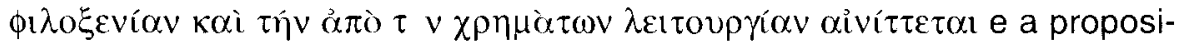

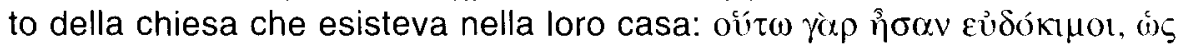

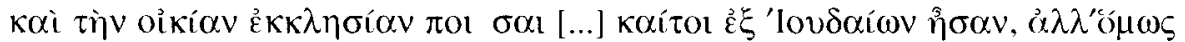

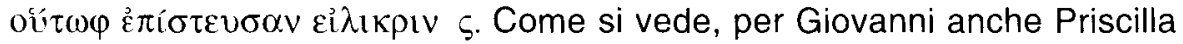
proveniva dal Giudaismo, sebbene le fonti neotestamentarie non lo dicano, bensi affermino solo di Aquila che era un Giudeo. Seguono le lodi di Priscilla, che possedette le autentiche ricchezze e gli ornamenti di una donna, ossia le virtù, e infine un'esaltazione di entrambi: $\beta \lambda \varepsilon \dot{\pi} \varepsilon \alpha \dot{v} \tau \vee \tau o ̀ v$

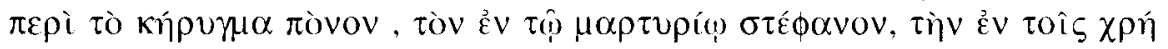

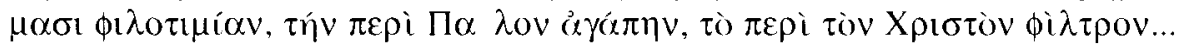

Ad Aquila e Priscilla sono collegati dalla tradizione due luoghi della storia cristiana di Roma, il titulus Priscae, ovvero la chiesa di Prisca sull'Aventino, che sarebbe sorta nel sito dell'antica domus ecclesiae dei due sposi, e le catacombe di Priscilla. Cercheremo di vedere quanto possano esser fondate queste identificazioni.

\section{IL TITULUS PRISCAE}

Se, come vedremo meglio, secondo il De Rossi l'eponima delle catacombe di Priscilla era la madre del senatore Pudente vissuto in età apostolica, lo stesso studioso ha supposto che anche tra la chiesa di S. Prisca e i Cornelii Pudentes intercorressero stretti legami. Infatti un diploma in bronzo (C/L VI n. 1454=31659) ritrovato nel 1776 presso $S$. Prisca e datato al 13 aprile 222 risulta deliberato dal concilium conventus Clunieni a C. Marius Pudens Cornelianus - personaggio di rango senatorio che la città aveva scelto come patronus- e secondo il medesimo De Rossi 7 dimostra che il sito dell'odierna chiesa apparteneva al principio del III secolo ai Cornelii Pudentes. Lo studioso riteneva altresi che $C$. Marius Pudens Cornelianus si chiamasse in origine C.Cornelius Pudens e che fosse entrato per adozione nella gens Maria ${ }^{8}$, ma fu confutato da Leclercq ${ }^{9}$, per cui non è detto che Cornelianus fosse né un adottato né,

De Rossi, Priscilla e gli Acilii Glabriones, pág. 126.

\& Eiusd. Della casa d'Aquila sull'Aventino, pág. 46. 
in origine, un Cornelius Pudens e che avanza piuttosto, con il Duchesne, l'ipotesi di una parentela tra gli Acilii Glabriones del cimitero di Priscilla con i Cornelii sulla base dell'uso del nome Cornelius -in virtù della discendenza in linea materna- da parte dell'Acilius Glabrio che fu console nel 152, e dunque dell'esistenza, al principio del II secolo d.C., di Cornelii Prisci ${ }^{10}$. Per il De Rossi la Prisca a cui la chiesa è dedicata è senz'altro la moglie di Aquila; egli ipotizza perfino che ella fosse liberta degli Acilii Glabriones, forse della stessa Priscilla madre di Pudente che egli, come vedremo, considera eponima delle catacombe. II De Rossi infatti collega ad Aquila e Prisca sia la chiesa sull'Aventino (il titulus Priscae) sia il cimitero di Priscilla, quello dell'omonima catacomba. Sepolta nel cimitero di proprietà della famiglia, Priscilla sarebbe abitata insieme con il marito in una casa concessale dagli stessi Glabriones --ai quali era ritornata dopo la morte degli sposi- nel cui sito sull'Aventino nel IV secolo sarebbe stata costruita una chiesa ${ }^{11}$. I rinvenimenti di vetri con effigi apostoliche presso S. Prisca confermò il De Rossi nella sua ipotesi che la chiesa sia fondata sulla casa dei due sposi ${ }^{12}$. Lo stesso De Rossi identifica gli Aquila e Prisca di cui il Liber Pontificalis (II 115-116 Duchesne) attesta che le reliquie furono trasferite sotto Leone IV (847-855) nella chiesa dei Quattro Coronati con i due sposi amici di s. Paolo e ipotizza che i corpi fossero trasferiti dalle suddette catacombe di Priscilla ${ }^{13}$, dove, come vedremo, furono effettivamente sepolti un Aquila ed una Prisca. Già nel Liber del resto i due sono identificati con i collaboratori di s. Paolo: Aquilae atque Priscillae, uti Actus Apostolorum testantur, quondam co-

9 Prisque, col. 1883.

10 Un Cornelius Priscus fu console nel 103 e proconsole d'Asia nel 120-121. Sulla base delle precedenti considerazioni, il Leclercq conclude che è possibile, ma non dimostrabile, una parentela tra gli Acilii Glabriones e i Cornelii Pudentes.

Ibid. e Priscilla e gli Acilii Glabriones, págs. 131-133.

2 Della casa d'Aquila sull'Aventino, pág. 88. Tutte le attestazioni archeologiche, iconografiche ed epigrafiche della connessione tra la chiesa di s. Prisca e la presenza di s.Pietro durante il suo soggiorno a Roma sono tarde e prive di valore documentario: un capitello forse di epoca antonina con un'epigrafe del XII-XIII secolo: bactismu(m) $s(a n) c(t) i$ Petri, un mosaico della medesima epoca situato - almeno fino a quando la chiesa non fu restaurata nel secolo scorso- nella cripta della chiesa e ratfigurante s. Pietro; un'epigrafe in versi scritta nel IX secolo sulla porta d'ingresso della chiesa che ricorda il presunto soggiorno di Pietro sull'Aventino (è l'iscrizione che abbiamo già ricordato e che comincia con haec domus est Aquilae seu priscae virginis almae); un'epigrafe del XV secolo nel coro, a sinistra dell'altare: Montis Aventini nunc facta est gloria maior/ unius veri relligione Dei. / Precipue ob Priscae quod cernis nobile templum / quod priscum merito par siib nomen habet. / Nam petrus id coluit populos dum saepe doceret, / dum faceret magno sacraque saepe Deo. / Dum quos Faunorum fontis deceperat error, / hic melius sacra purificaret aqua. Cfr. Leclercq, Prisque, col. 1882

13 G.B. DE Rossı, Escavazioni e scoperte nel cimitero di Priscilla, "Bullettino di Archeologia cristiana" 1880 , pág. 51 . 
mitum Pauli: evidentemente nel IX secolo, rinvenendo le epigrafi con i nomi di Aquila e Prisca, si pensò che fossero le due persone in questione. Questa identificazione però non sembra poggiare su basi incontrovertibili.

II diploma bronzeo di Corneliano non implica necessariamente ${ }^{14}$ che il titulus sanctae Priscae sia sorto nel IV sec. sulla dimora dei Cornelii Pudentes del III sec. e su quella, del I sec., di Aquila e Priscilla: nei documenti più antichi, dal V all'VIII secolo, si parla per la chiesetta semplicemente di titulus sanctae Priscae, senza alcuna menzione di Aquila ${ }^{15}$, il cui nome compare solo successivamente, nella vita di papa Leone III (^ 816) nel Liber Pontificalis (II 20 Duchesne), in un sermone de sanctis Aquila et Prisca, dove si situa sull'Aventino la loro dimora (cod. Vat. 1193), negli Atti di Prisca vergine e martire (AASS, ian., II, 551-552) e in un documento tardo, datato dal medesimo De Rossi al XII secolo, che menziona la $a b$ batia sanctorum Priscae et Aquilae, nonché nell'iscrizione databile secondo lo stesso ${ }^{16}$ all'VIII-IX secolo e posta sull'architrave della porta d'ingresso della chiesa sull'Aventino: haec domus est Aquilae seu Priscae virginis almae (ICVR II, 1, p. $443 \mathrm{n}$. 165). In quest'ultimo caso è evidente la confusione tra Prisca sposa di Aquila e s. Prisca Vergine.

A mio avviso è opportuno estendere dunque l'indagine al campo agiografico e considerare la presenza di un'altra Prisca, non associata ad Aquila: come dicevamo, infatti, nei documenti più antichi relativi al titulus Priscae non è mai fatta menzione di Aquila. Fonte importante sono allora gli Acta Sactorum, II, Iulii, Venezia 1752, pp. 534-37, De SS aquila et Priscilla coniugibus, forte in Asia Minore, Sylloge Historica, che pongono uno stretto legame tra Aquila e Prisca, da un lato, e s. Prisca Vergine e Martire, dall'altro lato, distinguendo però al contempo molto nettamente le due Sante omonime. Aquila e Prisca erano venerati a Roma da tempo antichissimo e gli Actus $S$ Priscae virginis et martyris narrano come il corpo della beata Prisca Vergine, Martire nel III secolo sotto l'imperatore Claudio

\footnotetext{
Come fa notare il Leclercq, Prisque, col. 1882.

Nel 499: Dominicus presbyter tituli Sanctae Priscae (Monumenta Germaniae Historica, Auct. Antiq., XII, p. 413 n. 45), nel 595: Maurus presbyter Sanctae Priscae; nel 721: Joannes presbyter sanctae Romanae ecclesiae tituli Sanctae Priscae; nel 745: Dominicus humilis presbyter sanctae Romanae ecclesiae tituli Sanctae Priscae; in un'epigrafe del V sec.: AVRelius TITuli PRISCAE e in una successiva: LOCVS ADEODATI PRESBYTERI TITVLI PRISCAE (L. Duchesne, Les titres présbiteraux et les diaconies, "Mél. d'Archéologie et d'Histoire" 7 (1887), p. 226). Anche il Liber Pontificalis sotto papa Adriano (772-795) parla solo di Prisca per it titulus e non di Aquila (1 pág. 501 Duchesne); cfr. Leclercq, Prisque, col. 1885.

16 Rispettivamente in Della casa d'Aquila sull'Aventino, pág. 45 e ibid.; Priscilla e gli Acilii Glabriones, p. 128.
} 
II, fu recato nella città di Roma con inni e cantici spirituali nella Chiesa dei ss. Martiri Aquila e Prisca. Dal documento risulta dunque che nel III secolo i due -ritenuti martiri come abbiamo visto che li ritiene Giovanni Crisostomo- erano già venerati, che avevano una loro chiesa e che in questa la santa martire, forse in virtù dell'omonimia, forse per un effettivo legame familiare, venne tumulata: se la chiesa in questione è, come sembra, quella dell'Aventino, parrebbe di dover supporre una sua duplice connessione con Prisca, da un lato con la moglie di Aquila, dall'altro con la Vergine del III secolo. La notizia degli Actus risulta quindi armonizzatrice di due tradizioni, che collegano la chiesetta ciascuna con una delle due Prisca. Non possiamo tuttavia considerare gli Actus alla stregua di una fonte storica pienamente fededegna, cosicché non è certo che la chiesa in cui s. Prisca Vergine fu recata fosse già dedicata ad Aquila e Prisca, tanto più che i riferimenti alla titolare della chiesa, come abbiamo visto, non menzionano mai Aquila fino a data tarda e che solo nella prima metà del IX secolo abbiamo notizia, come dicevamo, del trasferimento dei corpi di un Aquila e di una Prisca nella Chiesa dei Quattro Coronati; e non è un caso che sia proprio a partire dal IX secolo che la chiesa di s. Prisca prende ad essere denominata "di Aquila e di Prisca". I Bollandisti quindi giustamente distinguono in ogni caso la moglie di Aquila dalla vergine romana che patì martirio sotto Claudio II e la cui festa è ancor oggi il 18 gennaio (AASS, lan., II, 551-552): sembra probabile che sia questa la Prisca cui va collegata la chiesa dell'Aventino.

Dunque risulta problematico istituire un legame tra la Priscilla moglie di Aquila del titulus sanctae Priscae dell'Aventino, sebbene oggi l'identificazione del titulus con la primitiva dimora di Aquila e Prisca sia rivalutata da Giovanni Ricciardi, che ricorda come gli scavi condotti sul sito del titulus Priscae dal 1933 al 1966 abbiano portato alla scoperta di due case risalenti rispettivamente al II sec. d.C., con un mitreo, e al I d.C. ${ }^{17}$. Questo attesta indubbiamente l'antichità del sito e la sua importanza, e mostra come la chiesa della Prisca del III secolo sorgesse su edifici precedenti -e non è escluso in assoluto che uno potesse essere la antica casa di Aquila e Prisca, come sostengono gli Actus--, ma non dimostra in alcun caso, a mio parere, che si debba necessariamente identificare il sito stesso con la domus ecclesiae dei collaboratori di s. Paolo ${ }^{18}$.

\footnotetext{
17 S. FALASCA-G. RICCIARDI, O Roma Felix. Luoghi della memoria cristiana a Roma, Roma 1999, págs. 27-28.

18 Vale anche la pena di ricordare a questo proposito che il C. Marius Pudens dell'epigrafe bronzea del III secolo reca lo stesso nome del senatore romano che secondo gli Atti apocrifi di s.
} 


\section{LE CATACOMBE DI PRISCILLA E GLI ACILI/ GLABRIONES}

Sembra invece sicura la connessione tra le catacombe di Priscilla, da un lato, e gli Acilii Glabriones dall'altro, come ammette la Sordi ${ }^{19}$, seguita oggi dal Saxer, che sostiene ${ }^{20}$ : «uno dei nuclei della catacomba omonima deve loro [sc. agli Acilii Glabriones] senza dubbio la sua esistenza". Nella famiglia degli Acilii Glabriones in effetti il nome di Priscilla, eponima della catacomba, era frequente; esso compare molte volte nelle iscrizioni dell'ipogeo del cimitero detto appunto di Priscilla ${ }^{21}$. Tra le iscrizioni dell'antico ipogeo della necropoli priscilliana sono individuabili infatti quelle di Acilia Priscilla clarissima (femina o puella) e di sua madre Aria Plaria Vera Priscilla, moglie di $M^{\prime}$ Acilius Glabrio, che fu console per la seconda volta nel 186; è attestata anche un'altra Priscilla, moglie di $M$ ' Acilius $V$..., nonché altri membri della famiglia degli Acilii Glabriones ${ }^{22}$. Inoltre il nome di PrisCILLA ACILIANA si legge in un'iscrizione di Marino (CIL XIV 2484) e va collegato alla figlia di $M$ ' Acilius Glabrio console nel 152 e marito di una Priscilla Vera secondo un'iscrizione di Pesaro ${ }^{23}$. A conferma della persistente diffusione del nome Priscilla in queste catacombe, ancora nel $V$ secolo, è attestata epigraficamente nelle medesime catacombe priscilliane una Priscilla in un'iscrizione che cita un prefetto probabilmente identificabile con il celebre Anicius Acilius Glabrio Faustus, che fu prefetto di Roma ripetutamente sotto Valentiniano II e fu console ordinario nel 438. Data dunque la costante presenza di donne di nome Priscilla tra gli acilii Glabriones sepolti in questo cimitero, non è certamente necessario individuare nella Priscilla che dà il nome al cimitero stesso la sposa di Aquila, né sembra possibile avvalersi dell'argomento della somiglianza del nome di Aquila, Aculas, con il gentilizio Acilius, talora invocata in favore del collegamento tra Aquila e Priscilla, da

Pietro avrebbe ospitato quest'ultimo nella sua dimora sul vicus Patricius, dove oggi sorge la chiesa di S. Pudenziana, alie falde del Viminale. E vero che gli Atti apocrifi non sono certo una fonte storica attendibile; essi tuttavia possono aver serbato, tra le molte fantasie di cui sono infarciti, anche alcune notizie storiche, come ad esempio anche l'intervallo di anni tra la Crocifissione e l'inizio della predicazione: Sordi, 1 Cristiani, pág. 35.

19 Sordi, /l Cristianesimo e Roma, pág. 110.

20 Priscilla, col. 2905.

21 Su cui cfr. H. Leclercq, Priscille, cimetiere de, in DACL, XIV, 1, Paris 1948, coll. 1799-1874.

22 Questi i testi di alcune delle più rilevanti attestazioni: ACILIO GLABRIONI Fi/IO...; Manius

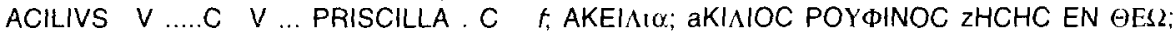

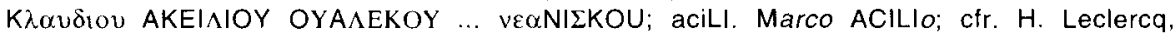
Glabriones, in DACL, VI, coll. 1275-1270, rispettivam. nn. 5300-5305.

23 Cfr. "Bullettino di archeologia cristiana" 1888, pág. $42 \mathrm{sg}$.: il De Rossi, che ha studiato l'iscrizione, sostiene che gli Acilii Veri citati da noi alla nota precedente e i cui nomi sono stati rinvenuti nell'ipogeo degli Acilii Glabriones sono i figli di questa coppia, anche se secondo C.L. Visconti e H. Leclercq potrebbero essere anche i loro nipoti (cfr. Leclercq Priscille, col. 1808). 
un lato, e, dall'altro, il cimitero di Priscilla. Sotto il nome di Priscilla tale cimitero risulta già menzionato più volte nel più antico catalogo dei cimiteri inserito nella Notitia e risalente almeno al $\mathrm{V}$ secolo (coemeterium Priscillae ad S. Silvestrum via Salaria).

Situato al terzo miglio della Via Salaria Nuova, l'ipogeo fu oggetto di scavi e studî sistematici a partire dal secolo scorso con G.B. De Rossi. L'importanza di questi rinvenimenti risiede soprattutto nella data particolarmente alta in cui essi si possono collocare, tra il primo secolo d.C. e la metà del secondo ${ }^{24}$ : certo, non sempre è facile determinare la datazione precisa delle epigrafi cristiane (come quelle che recano le diciture Marcianus hic dormit in pace, oppure Caelestina pax, o Zosime pax tecum, quest'ultima probabilmente già di età damasiana, o una con il nome Terentius preceduto dal chrismon, oppure ancora una che reca il più antico esempio di A e W nell'epigrafia funeraria cristiana, con Modestina A W) ${ }^{25}$ o, per converso, il carattere cristiano delle epigrafi più antiche ${ }^{26}$, ma in alcuni casi sembra di poter disporre di alcuni punti fermi: ad esempio, è

24 Per la cronologia delle varie parti del cimitero cfr. Leclercq, Priscille, col. 1818. Scriveva già il Duchesne, a pochi anni dalle esplorazioni del cimitero: "le cimetière de Priscille a contenu et contient encore, par centaines, des tombes dont le caractères archéologiques sont tels que nous n'hésitons pas à les faire remonter au temps de saint Justin, d'Hermas, même de saint Clément" ( $L$. Duchesne, Lettera a O. Marucchi, Roma, 12 luglio 1901, in "Nuovo Bullettino di Archeologia cristiana», 1901, págs. 113-118). Lo stesso Duchesne era dubitativo riguardo all'opportunità di identificare la Priscilla delle catacombe con la moglie di Aquila, ma si avvedeva bene che non ne rimane inficiata l'antichità del cimitero e il suo legame con gli Acilii Glabriones: “ll faut, je crois, être très réservé à l'endroit de conjectures qui rattacheraient la Priscille éponyme du cimetière à la femme du juif Aquila [...] mais, ceci mis à part, le cimetière de Priscille présente une antiquité telie que son développement connu n'est pas loin d'atteindre l'âge apostolique» (ibid.).

25 L'ultima è un'iscrizione posta su un loculo e contrassegnata con il n. 10534 dal Leclercq, Priscille, col. 1824, che ne offre anche il disegno. Un'altra iscrizione trovata dappresso e dipinta in colore rosso, sempre in un loculo, suona: SPIRItus tuus REQVESQVAT, mentre il nome del defunto è andato perduto: certamente si tratta di un'iscrizione cristiana, ma non è databile con precisione. Di estremo interesse sono poi gli esempi di carmi epigrafici offerti dall'epigrafia priscilliana, in cui il carme di Agape (sit vestrae mentis Agapes carae meminisse, / ut deus omnipotens Agapen in saecula servet) funge da modello per altri successivi, in particolare uno contenente il nome Marcia, cosi come l'epitafio di Abercio fu modello per quello di Alessandro (per l'epitafio di Abercio cfr. eventualmente il mio L'epitafio di Abercio: uno status quaestionis e alcune osservazioni, in corso di pubblicazione su "Aevum" 2000). II Leclercq, Priscille, col. 1823 suppone anche che vi fosse un modello comune sia per l'epitafio di Agape sia per quello di Marcia. Certamente cristiane ma difficilmente databili sono poi le iscrizioni come MARCIANVS HIC / DORMIT IN PACE.

${ }^{26}$ Ad es. è senz'altro del III secolo l'epigrafe EVSEBIAE FILIAE DVLCISSIMAE Quae VIXIT ANNIS / DVOBVS MENSE VNO DIEBVS XXIIII PARENTES, anche se non vi sono elementi decisivi per poter affermare che si tratti di un'iscrizione cristiana; databile all'ultimo quarto del II secolo è un'epigrafe greca di cui analogamente non si puó predicare il carattere cristiano: KHIA FOIBH/ TH FILANDRW KA/PITWN O CUNBIOC / KAI EAUTW; della seconda metà del II secolo è l'epigrafe presentata dal Leclercq sotto il n. 10532: AVRELIO BRACaro? filio? / dulcisSIMO VENVSTVs pater? cfr. Leclercq, Priscille, coll. 1815-1817. 
stato rinvenuto uno stampo doliare dell'epoca di Antonino Pio. I simboli stessi qui attestati - l'ancora, il pesce, la palma, le colombe, ma non ancora il monogramma di Cristo - sembrano condurre ad una data alta, cosi come le formule spes e pax tibi, ripetutamente presenti, e l'iscrizione TITVS FLA/VIVS FE/LICISSIMVS/ POSITVS EST, databile paleograficamente ed anche in base alla formula positus est, più antica di depositio, agli inizi del II secolo. L'uso dei tria nomina sembra inoltre indicare che il defunto apparteneva alla famiglia imperiale dei Flavi, probabilmente era un loro liberto. Questo non sorprende, se si pensa che alla fine del I secolo in seno alla gens Flavia c'erano illustri Cristiani, come Flavio Clemente e Flavia Domitilla, condannati per Cristianesimo da Domiziano, contemporaneamente ad Acilio Glabrione e con le medesime accuse di ateismo e devianza verso costumi giudaici. II particolare ha un'importanza capitale nel nostro discorso, come ora vedremo. Alcune iscrizioni certamente cristiane sono databili con sufficiente sicurezza, come quella del III secolo che presenta in cima una ghirlanda fiancheggiata da due colombe, una per lato, e recante la dicitura: SENTVS MERCVRIVS SIBI ET COIVGI SUAE I CENSORINAE AMANTIAE BENEMERENTI PACE ${ }^{27}$. Altri elementi datanti parrebbero venire dall'iconografia: le decorazioni pittoriche della cosiddetta capella Greca la fanno datare alla prima metà del II secolo ${ }^{28}$. Inoltre, un affresco di Maria con il Bambino sembra "non posteriore ai primi Antonini e forse alquanto anteriore" e databile comunque a prima della metà del II secolo ${ }^{29}$. Di poco più tarda ma significativa appare la formula Deo bolente [sic] / Felix / Ampliatus, in cui si trova un evidente adattamento della formula pagana diis volentibus e che sembra risalire al III secolo al più tardi o piuttosto, secondo il Leclercq ${ }^{30}$, al II. Per alcune iscrizioni antiche, in caratteri priscilliani e non lontane nel tempo da quella di Felix Ampliatus, nonostante la mancanza di formule esplicitamente cristiane, sembra molto probabile, in base ai nomi e ai contenuti, che siano di gente cristiana: in due infatti Paulus e Petrus sono usati come cognomina $e$ in una si loda che un giovane morì virgo (LVCRETIO PAVIo inFANTI DVLCIS/SIMO QVI BIXIT ANNO UNO MENS/HI DIES XVI LVCRETIVS

27 Cfr. Leclercq, Priscille, col. 1816.

28 H. Leclercq, Capella Greca, in DACL, II, Paris 1924, coll. 2084-2216; Eiusd. Priscille, coll. 1812-1813 per la descrizione dell'atrium (contemporaneo alla cappella stessa: probabilmente l'atrio accoglieva i fedeli e la cappella il clero) e dei rinvenimenti epigrafici quivi occorsi. L'analisi del Leclercq autorizza ad affermare quanto segue: "ses fresques autorisent à la dater de la première moitié du llème siècle, elle était donc de peu posterieure à la région cimétériale, de sorte que ce n'est pas le noyau du cimetière de Priscille, mais une annexe très ancienne" (Priscille, col. 1812).

${ }_{29}$ Cfr. la tavola in DACL, II, Paris 1924, col. 2476, tavola fuori testo; Leclercq, Priscille, col. 1811.

30 Leclercq. Priscille (cimetière de), col. 1824. 
EVTYCHES/ ET LVCRETIA MAXIMILLA PARENTES; AVREL PETRO FILio / DVLCSSIMO QVI Vixit annos ... / MENS VII VIRGO AUR M... / AEL DONATA PARENTES / PELAGIORVM).

Sembra assai probabile che il Cristianesimo sia entrato molto presto nella famiglia degli Acilii Glabriones, di cui il primo Cristiano sembra essere stato il senatore messo a morte da Domiziano per costumi giudaici, ateismo e combattimento con le fiere e menzionato da Giovenale (sat. IV) ${ }^{31}$, da Suet. Dom. 10 e da Dio Cass. LXVII 14: Baronio e Ruinart hanno interpretato per primi l'accusa di adesione a costumi giudaici, di ateismo e di combattimento con le fiere, che sottintendeva pratiche magiche, come una accusa di essere cristiano, prima ancora che G.B. De Rossi scoprisse nel 1888 l'ipogeo degli Acilii Glabriones nelle catacombe di Priscilla sulla via Salaria, anche se con iscrizioni del II-III secolo e non del I, come abbiamo visto ${ }^{32}$. Gli Acilii ivi contenuti sono comunque discendenti di quello messo a morte da Domiziano e che era cristiano. Infatti, a conferma del cristianesimo dell'Acilio Glabrione condannato da Domiziano sembra deporre, accanto al tipo stesso di accuse con cui egli fu mandato a morte secondo Svetonio e secondo Dione Cassio, il fatto che la sua morte sia deplorata da Giovenale nella medesima satira in cui egli forse allude al supplizio che, sempre a Roma e sempre sotto Domiziano, ebbe a subire un altro Cristiano, s. Giovanni evangelista, e in cui alla fine adombra i Cristiani, non senza un certo distacco, nei cerdones che contribuirono alla fine di Domiziano ${ }^{33}$. Se dunque già nel I secolo il Cristianesimo era presente con ogni probabilità nella famiglia degli Acilii Glabriones, nella cui proprietà si trova il cimitero di Priscilla, sembra che i contatti tra gli Acilii stessi e la comunità cristiana di Roma vadano fatti risalire molto indietro nel tempo. Divengono allora significativi due dati: sotto il pontificato di Leone IV nel cimitero di Priscilla fu trovata una serie di tombe, fra cui que-

${ }^{31}$ Sull'importanza della sat. IV di Giovenale come fonte si vedano A. Luisi, II rombo e la vestale, Bari 1998, e il mio La satira IV di Giovenale e il supplizio di s. Giovanni a Roma, di prossima pubblicazione su "Gerión".

32 Cfr. G.B. De Rossi, Lipogeo degii Acilii Glabriones nel cimitero di Priscilla, «Bull. di Archeol. Crist. 6 (1888-89), págs. 15-66; 103-133; dubbî sono sollevati dal Leclercq. Prisque, col. 18883-84; Bibliotheca Sanctorum, X 1113-1114; Dictionnaire d'Archéologie Chrétienne et de Liturgie I, 2854 2860; ibid. VI, coll. 1269-74; Dictionnaire d'Histoire et Geographie Ecclesiastique, I, 338-40; M. Sordi, La persecuzione di Domiziano, "Rivista di Storia della Chiesa in Italia" 14 (1960), págs. 7-13; P. Keretzes, The Jews, the Christians and the Emperor Domitian, "Vigiliae Christianae" 27 (1973), págs. 1-28; A. Amore, I martiri di Roma, Bologna 1966, págs. 50-66; P.-W., I, 1, 257; V. Saxer, Acilio Glabrione, in Dizionario Patristico e di Antichità Cristiane, I, Casale Monferrato 1983, col. 36.

${ }_{33} \dot{E}$ un'ipotesi che, sia pur con ogni cautela, ma comunque sulla base di molti indizi ricavabili dalla satira stessa, avanzo nel già cit. La satira IV di Giovenale.

34 Leclercq, Priscille, col. 1801. 
Ila di un certo Aquila e quella di una certa Prisca ${ }^{34}$. Certamente la coincidenza dei nomi non è probante, però non è escluso in linea assoluta che i due possano essere identificati con i collaboratori di s. Paolo. La loro sepoltura nel cimitero di Priscilla potrebbe suggerire un eventuale contatto, sin dall'inizio, tra i collaboratori di Paolo e la famiglia degli Acilii Glabriones che ben presto, sotto Domiziano, come probabilmente anche quella dei Flavii Clementes, avrebbe dato un senatore martire. Nel III secolo, poi, secondo il ms. di Epternach del Martyrologium Hieronymianum la Martire Prisca - di cui abbiamo già ricordato la notizia, sospetta, della deposizione in una chiesa dedicata alla memoria di Aquila e Prisca- fu sepolta sulla via Salaria e, secondo gli Itineraria, proprio al terzo miglio, dove si trovavano appunto le catacombe di Priscilla. Ma l'eponima delle catacombe stesse non è probabilmente né la moglie di Aquila né la santa Martire: nel Liber Pontificalis, nella vita di papa Marcello, che visse al principio del IV secolo, si ricorda: rogavit quandam matronam nomine Priscillam et fecit cymiterium (Novellae) via Salaria: Marcello avrebbe sepolto il suo predecessore Marcellino nelle catacombe di Priscilla (Marcellus presbyter collegit noctu corpora et sepelivit in via Salaria in cymiterio Priscillae): sembra la prima volta che un papa viene sepolto in questo cimitero. L'eponima delle catacombe è dunque una matrona romana, anche se non certo quella dell'epoca di Marcello, ma una ben precedente; secondo il De Rossi si tratta addirittura della madre del senatore Pudente, il medesimo che secondo la tradizione avrebbe offerto ospitalità all'apostolo Pietro e il cui ricordo è associato ai figli Pudente, Pudenziana e Prassede ${ }^{35}$. Questa supposizione non sembra però trovare elementi di sicura conferma; tuttavia appare probabile che l'eponima sia una delle matrone degli Acilij Glabriones, una famiglia in cui abbiamo visto frequente il nome di Priscilla. Dunque il cimitero di Prisca fu creato nei possedimenti degli Acilii, forse già in età apostolica: non è escluso che sia i collaboratori di Paolo sia soprattutto la Martire Prisca possano essere stati sepolti qui, nelle proprietà dei nobili cristianizzati Acilii Glabriones. Ilaria Ramelli, via Guerra 2, 29100 Piacenza.

35 G.B. De Rossi, Priscilla e gli Acilii Glabriones, “Bull. Arch. Cristiana» 1889, págs. 103-133. 